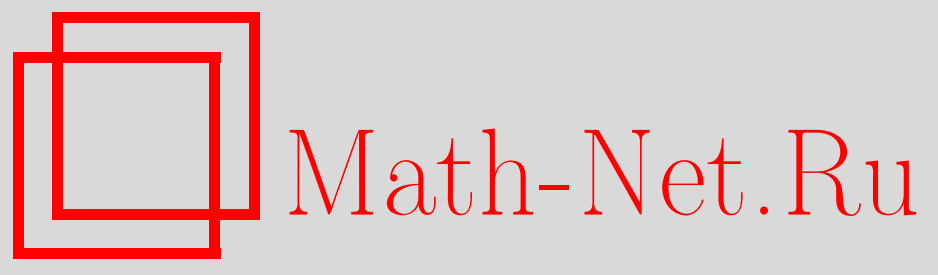

И. Э. Зверович, Сильные $k$-раскраски графов, Дискрет. матем., 2001, том 13, выпуск 1, 78-89

DOI: https://doi.org/10.4213/dm279

Использование Общероссийского математического портала Math-Net.Ru подразумевает, что вы прочитали и согласны с пользовательским соглашением http://www.mathnet.ru/rus/agreement

Параметры загрузки:

IP : 3.82 .47 .9

26 апреля 2023 г., 17:41:59

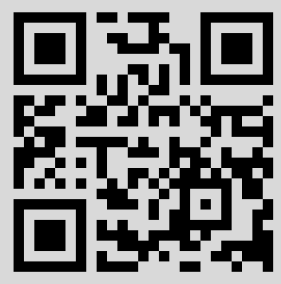


УДК 519.1

\title{
Сильные $k$-раскраски графов
}

\author{
(c) 2001 г. И. Э. Зверович
}

\begin{abstract}
Правильная $k$-раскраска $C_{1}, \ldots, C_{k}$ графа $G$ называется сильной, если для любой вершины $u \in V G$ существует индекс $i \in\{1, \ldots, k\}$ такой, что $u$ смежна с каждой вершиной класса $C_{i}$. В этой работе рассмотрен класс $S(k)$ сильно $k$-раскрашиваемых графов и показано, что задача распознавания $S(k)$ является NP-полной при любом $k \geqslant 4$, а при $k=3$ - полиномиально разрешимой. Мы даем характеризащию класса $S(3)$ в терминах запрещенных порожденных подграфов и решаем проблему единственности сильной 3-раскраски.
\end{abstract}

Мы придерживаемся, в основном, терминологии книг $[1,3]$. Хорошо известно, что задачи $k$-раскраски (разбиения множества вершин графа на $k$ подмножеств с заданными наследственными свойствами порожденных подграфов) часто являются сложными уже при малых значениях $k$. Так, задача распознавания $k$-раскрашиваемых графов (в обычном смысле) является NP-полной при $k \geqslant 3$ [1]. Для графов, имеющих $(k, l)$-раскраску (то есть разбиение на $k$ независимых множеств и $l$ клик), задача распознавания NP-полна при $\max \{k, l\} \geqslant 3$ (см. [2]).

Однако, введение дополнительных условий на множества ребер между цветными классами позволяет получить в некоторых случаях полиномиально распознаваемые классы. Так, можно показать, что все классы графов, имеющих $l$-ограниченную $k$-раскраску, могут быть охарактеризованы конечным числом запрещенных порожденных подграфов, что влечет полиномиальную распознаваемость таких классов.

В этой работе мы рассмотрим класс $S(k)$ сильно $k$-раскрашиваемых графов и покажем, что задача распознавания $S(k)$ является NP-полной при любом $k \geqslant 4$, а при $k=3$ полиномиально разрешимой. Кроме того, мы дадим характеризацию класса $S(3)$ в терминах запрещенных порожденных подграфов и решим проблему единственности сильной 3-раскраски.

Запись $u \sim v$ (соответственно, $u \nsim v$ ) означает, что вершины $u$ и $v$ смежны (соответственно, не смежны). Окружением вершины $u$ графа $G$ называется множество $N(u)=\{u \in V G: u \sim v\}$. Если $X, Y \subseteq V G$ и $X \cap Y=\varnothing$, то $X \sim Y$ (соответственно, $X \nsim Y$ ) означает, что $x \sim y$ (соответственно, $x \nsim y$ ) для любых $x \in X$ и $y \in Y$. При $X=\{x\}$ мы будем писать $x \sim Y$ и $x \nsim Y$ вместо $\{x\} \sim Y$ и $\{x\} \nsim Y$. Подграф графа $G$, порожденный множеством $X=\left\{x_{1}, \ldots, x_{n}\right\} \subseteq V G$, обозначается через $G(X)$ или $G\left(x_{1}, \ldots, x_{n}\right)$.

Правильная $k$-раскраска $C_{1}, \ldots, C_{k}$ графа $G$ называется сильной, если для любой вершины $u \in V G$ существует индекс $i \in\{1, \ldots, k\}$ такой, что $u \sim C_{i}$. Обозначим через $S(k)$ класс всех графов, имеющих сильную $k$-раскраску. Ясно, что $S(1)=\varnothing$ и $S(2)$ есть множество всех полных двудольных графов (наследственный 


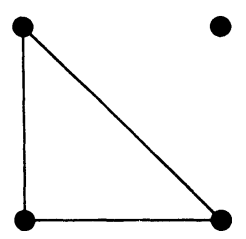

$G_{1}$

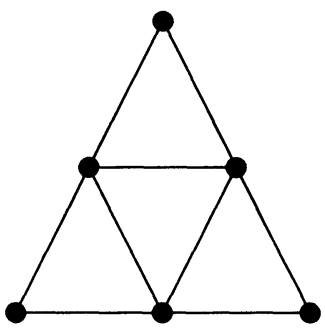

$G_{5}$

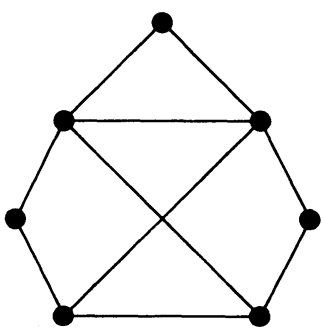

$G_{8}$

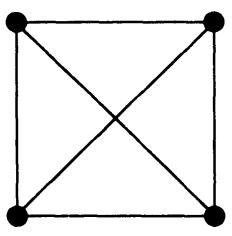

$G_{2}$

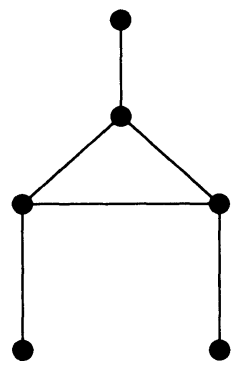

$G_{3}$

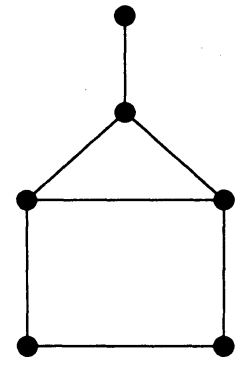

$G_{4}$

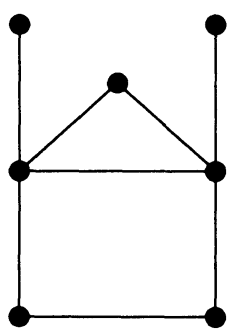

$G_{6}$

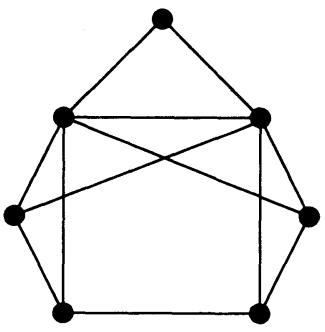

$G_{9}$

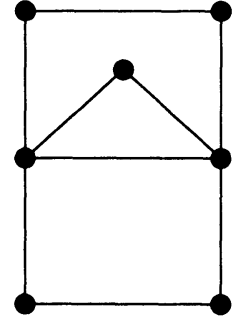

$G_{7}$

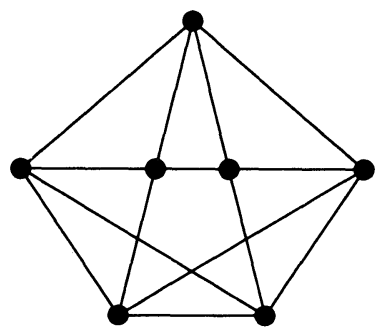

$G_{10}$

Рис. 1. Некоторые минимальные запрещенные порожденные подграфы для класса $S(3)$

класс, определяемый двумя запрещенными порожденными подграфами $K_{1} \cup K_{2}$ и $\left.K_{3}\right)$. Очевидно, что при любом $k \geqslant 3$ класс $S(k)$ также является наследственным и, следовательно, допускает характеризацию в терминах запрещенных порожденных подграфов.

Теорема 1. Граф $G$ принадлежит $S(3)$ тогда $u$ только тогда, когда он не содержит графов $G_{1}, \ldots, G_{10}$ (рис. 1) и простых циклов $C_{2 n+1}, n \geqslant 2$, в качестве порожденных подграфов.

Доказательство. Докажем необходимость.

Лемма 1. Если $H-$ двудолънъй граф, то $H \in S(3)$. 

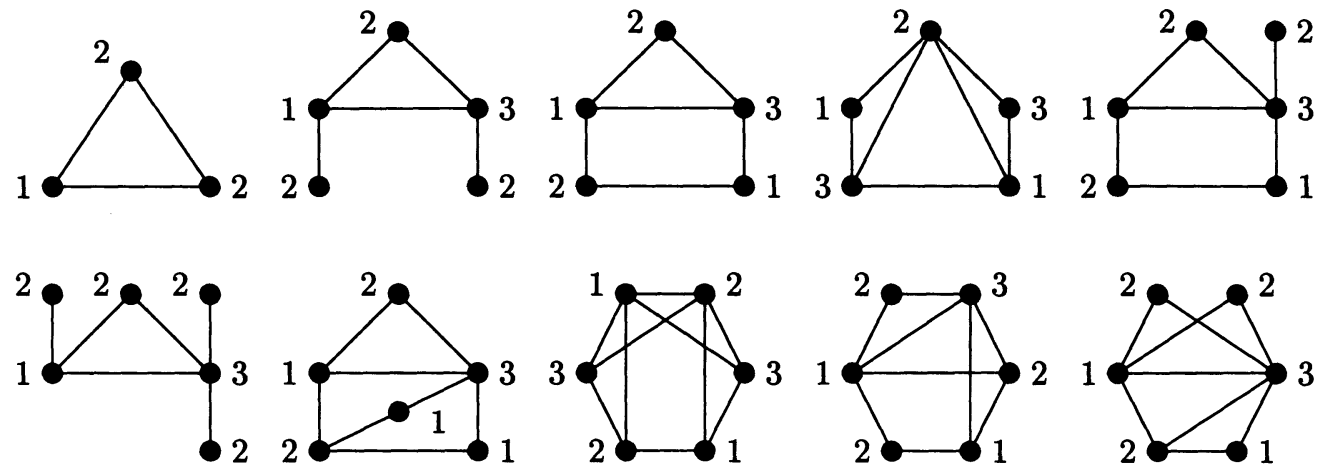

Pис. 2.

Доказательство. Пусть $C_{1} \cup C_{2}=V H$ есть разбиение на доли графа $H$. Тогда $C_{1}, C_{2}, C_{3}=\varnothing$ является сильной 3-раскраской графа $H$.

Покажем, что $H=C_{2 n+1} \notin S(3)$ при $n \geqslant 2$. Пусть $V H=\left\{v_{1}, \ldots, v_{2 n+1}\right\}$ (вершины перечислены в порядке обхода цикла). Пусть $C_{1}, C_{2}, C_{3}$ - сильная 3-раскраска графа $H$. Можно считать, что $v_{1} \in C_{1}$ и $v_{2} \in C_{2}$. Каждая вершина $v_{i}, 4 \leqslant i \leqslant 2 n$, не смежна с вершинами $v_{1}$ и $v_{2}$, поэтому $v_{i} \in C_{3}$. Так как граф $H$ не двудольный, то хотя бы одна из вершин $v_{3}$ или $v_{2 n+1}$ принадлежит $C_{3}$. В силу симметрии можно считать, что $v_{3} \in C_{3}$. Так как $v_{3}$ не смежна с $v_{1} \in C_{1}$ и $v_{2 n+1}$, то $v_{2 n+1} \notin C_{2}$. Следовательно, $v_{2 n+1} \in C_{3}$. Так как $v_{1}$ не смежна с $v_{3} \in C_{3}$ и $v_{4}$, то $v_{4} \notin C_{2}$. Поэтому $v_{4} \in C_{1}$. Тогда $v_{2} \in C_{2}$ не смежна с вершинами $v_{4} \in C_{1}$ и $v_{2 n+1} \in C_{3}$. Пришли к противоречию. Итак, простые циклы $C_{2 n+1}$ не принадлежат $S(3)$ при $n \geqslant 2$.

Непосредственно проверяется, что графы $G_{1}, \ldots, G_{10}$ (рис. 1 ) не имеют сильной 3 -раскраски. При удалении любой вершины из графов $G_{1}, \ldots, G_{10}$ и $C_{2 n+1}, n \geqslant 2$, получается либо двудольный граф, (который по лемме 1 принадлежит $S(3)$ ), либо один из графов, показанных на рис. 2 (которые имеют сильную 3-раскраску). Итак, графы $G_{1}, \ldots, G_{10}$ и $C_{2 n+1}, n \geqslant 2$, являются минимальными запрещенными порожденными подграфами для класса $S(3)$.

Докажем достаточность. Пусть граф $G$ не содержит порожденных подграфов $G_{1}, \ldots, G_{10}$ и $C_{2 n+1}, n \geqslant 2$. Вершина называется доминирующей, если она смежна со всеми другими вершинами графа.

Лемма 2. Если граф $G$ содержит доминирующую вершину $u$, то $G \in S(3)$ тогда и только тогда, когда граф $G-$ и является двудолъным.

Доказательство. Докажем необходимость. Пусть $C_{1}, C_{2}, C_{3}-3$-раскраска графа $G$. Можно считать, что $C_{1}=\{u\}$. Тогда $G-u$ имеет 2-раскраску $C_{2}, C_{3}$.

Докажем достаточность. Пусть $C_{2}, C_{3}$ есть разбиение на доли графа $G-u$. Тогда $C_{1}=\{u\}, C_{2}, C_{3}$ является сильной раскраской графа $G$.

Продолжим доказательство теоремы. Если в $G$ есть доминирующая вершина $u$, то граф $G-u$ не содержит треугольников (так как $G$ не имеет порожденного подгра$\left.\phi а G_{2}\right)$. Кроме того, все циклы $C_{2 n+1}, n \geqslant 2$, по условию не являются порожденными подграфами графа $G-u$. По теореме Кенига граф $G-u$ является двудольным. По 

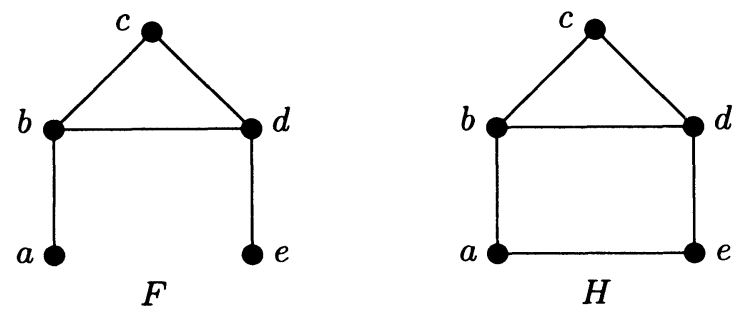

Рис. 3. Графы $F$ и $H$

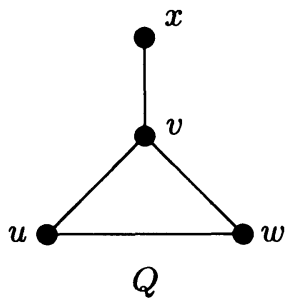

Рис. 4. Граф $Q$

лемме 2 справедливо включение $G \in S(3)$. Поэтому в дальнейшем будем считать, что в $G$ нет доминирующих вершин.

Вершины $u$ и $v$ называются раздвоенными, если $N(u)=N(v)$. В частности, раздвоенные вершины не смежны.

Лемма 3. Если и $и$ я являются раздвоенными вершинами графа $G$, то $G \in S(3)$ тогда и толъко тогда, когда $G-u \in S(3)$.

Доказательство. Необходимость вытекает из наследственности графа $S(3)$.

Докажем достаточность. Пусть $C_{1}, C_{2}, C_{3}$ является сильной 3-раскраской графа $G-u$, причем $u \in G_{1}$. Легко видеть, что $G_{1} \cup\{u\}, C_{2}, C_{3}$ является сильной 3-раскраской графа $G$.

В силу леммы 3 можно считать, что граф $G$ не имеет раздвоенных вершин.

Лемма 4. Если граф $G$, не имеющий порожденных подграфов $G_{1}, \ldots, G_{10} u C_{2 n+1}$, $n \geqslant 2$, не содержит доминирующих и раздвоенных вершин, то либо $G$ является двудольным, либо по крайней мере один из графов $F$ или $H$, показанных на рис. 3 , является порожденным подграфом графа $G$.

Доказательство. Пусть $G$ не является двудольным. Тогда $G$ содержит порожденный нечетный простой цикл $C=C_{2 n+1}$. При $n \geqslant 2$ получаем противоречие с условием. Поэтому $C=C_{3}$. Пусть $V C=\{u, v, w\}$.

Рассмотрим максимальное независимое множество $I$, содержащее вершину $u$. Так как $u$ не является доминирующей, то $|I| \geqslant 2$. Если существует вершина $x \in I \backslash\{u\}$, не смежная с одной из вершин $v, w$ (пусть $x \nsim w$ ), то $x \sim v$ (иначе $\{u, v, w, x\}$ порождает $\left.G_{1}\right)$ и мы получаем порожденный подграф $Q$, показанный на рис. 4. 
В противном случае произвольная вершина $x \in I \backslash\{u\}$ смежна с $v$ и $w$. Так как $u$ и $x$ не являются раздвоенными, существует вершина $y$, не смежная с одной из них и смежная с другой. В силу симметрии можно считать, что $y \sim u$ и $y \nsim x$. Так как $\{v, w, x, y\}$ не порождает $G_{1}$, можно считать, что $y \sim u$. Так как $\{u, v, w, y\}$ не порождает $G_{2}$, можно считать, что $y \nsim w$. Тогда множество $\{u, v, x, y\}$ порождает граф $Q$.

Итак, мы показали, что граф $G$ содержит порожденный подграф $Q$. (Обозначения вершин см. на рис. 4). Рассмотрим теперь максимальное независимое множество $J$, содержащее вершину $v$. Так как $v$ не является доминирующей, то $|J| \geqslant 2$. Если существует вершина $z \in J \backslash\{v\}$, не смежная хотя бы с одной из вершин $u, w$ (пусть $z \nsim u$ ), то $z \sim w$ (иначе $\{u, v, w, z\}$ порождает $G_{1}$ ) и множество $\{u, v, w, x, z\}$ порождает один из графов $F$ или $H$, что и требуется. В противном случае, произвольная вершина $z \in J \backslash\{v\}$ смежна с обеими вершинами $u$ и $w$. Так как $\{u, w, x, z\}$ не порождает $G_{1}$, то $u \sim z$. Далее, вершины $v$ и $z$ не являются раздвоенными, то есть существует вершина $t$, не смежная с одной из них и смежная с другой. В силу симметрии можно считать, что $t \sim v$ и $t \nsim z$. Как и выше, из отсутствия $G_{1}$ и $G_{2}$ следует, что $t$ смежна ровно с одной вершиной множества $\{u, w\}$. Пусть $t \sim u$ и $t \nsim w$. Тогда множество $\{u, w, x, z, t\}$ порождает $F$ или $H$.

Если граф $G$ является двудольным, то $G \in S(3)$ по лемме 1 . В противном случае, по лемме 4 граф $G$ содержит один из графов $F$ или $H$ в качестве порожденного подграфа.

Лемма 5. Если граф $G$ удовлетворяет условиям леммы 4 и содержит порожденный подграф $F$ (рuс.3), то $G \in S(3)$.

Доказательство. Если $G=F$, то $G \in S(3)$. В противном случае существует вершина $u \in V G \backslash V F$. Зафиксируем обозначения вершин подграфа $F$, указанные на рис. 3. Легко проверить, что $F$ имеет единственную (с точностью до переименования цветных классов) сильную 3 -раскраску $C_{1}, C_{2}, C_{3}$. Будем считать, что $C_{1}=\{b\}$, $C_{2}=\{a, c, e\}$ и $C_{3}=\{d\}$.

Покажем, что эта раскраска порождает единственную сильную 3-раскраску всего графа $G$. В табл. 1 показаны все 32 варианта для подграфа, порожденного множеством $V F \cup\{u\} ; 1$ (соответственно, 0 ) означает, что $u$ смежна (соответственно не смежна) с вершиной, указанной в заголовке столбца, а * означает, что в соответствующей позиции может быть как 0 , так и 1.

Итак, цвет каждой вершины $u \in V G \backslash V F$ определяется в соответствии с табл. 1 однозначно, то есть раскраска $C_{1}, C_{2}, C_{3}$ графа $F$ определяет разбиение $V G$ на три класса, которые мы также будем обозначать $C_{1}, C_{2}, C_{3}$.

Покажем, что $C_{1}$ порождает пустой граф. Допустим, что существуют смежные вершины $u, v \in C_{1}$. Ясно, что $u, v \notin V F$. Из табл. 1 видно, что обе вершины $u$ и $v$ смежны с вершинами $a$ и $d$, но не смежны с $b$. Так как $\{a, e, u, v\}$ не порождает $G_{1}$, то $e$ смежна с одной из вершин $u, v$. Пусть $e \sim v$. Так как $\{d, e, u, v\}$ не порождает $G_{2}$, то $e \nsim u$.

Если $c \nsim u$ и $c \nsim v$, то $\{a, c, u, v\}$ порождает $G_{1}$; если $c \nsim u$ и $c \sim v$, то $V F \cup\{u, v\}$ порождает $G_{9}$; если $c \sim u$ и $c \nsim v$, то $\{a, b, c, e, u, v\}$ порождает $G_{4}$; если $c \sim u$ и $c \sim v$, то $\{c, d, u, v\}$ порождает $G_{2}$.

Итак, во всех случаях получаем противоречие. Поэтому $C_{1}$ порождает пустой граф. В силу симметрии, $C_{3}$ также порождает пустой граф. 
Таблица 1.

\begin{tabular}{llllll}
\hline \hline$a$ & $b$ & $c$ & $d$ & $e$ & Запрещенный порожденный подграф, или класс вершины $u$ \\
\hline$*$ & 0 & 0 & 0 & $*$ & $G(b, c, d, u)=G_{1}$ \\
1 & 1 & 0 & $*$ & 0 & $G(a, b, e, u)=G_{1}$ \\
0 & $*$ & 0 & 1 & 1 & $G(a, d, e, u)=G_{1}$ \\
$*$ & 1 & 1 & 0 & 0 & $G(b, c, e, u)=G_{1}$ \\
0 & 0 & 1 & 1 & $*$ & $G(a, c, d, u)=G_{1}$ \\
$*$ & 1 & 1 & 1 & $*$ & $G(b, c, d, u)=G_{2}$ \\
0 & 0 & 1 & 0 & 0 & $G(a, b, c, d, e, u)=G_{3}$ \\
0 & 0 & 1 & 0 & 1 & $G(a, b, c, d, e, u)=G_{4}$ \\
1 & 0 & 1 & 0 & 0 & $G(a, b, c, d, e, u)=G_{4}$ \\
1 & 1 & 0 & 1 & 1 & $G(a, b, c, d, e, u)=G_{5}$ \\
1 & 0 & 1 & 0 & 1 & $G(a, b, d, e, u)=G_{5}$ \\
1 & 0 & $*$ & 1 & $*$ & $u \in C_{1}$ \\
$*$ & 1 & $*$ & 0 & 1 & $u \in C_{3}$ \\
0 & 0 & 0 & 1 & 0 & $u \in C_{2}$ \\
0 & 1 & 0 & 0 & 0 & $u \in C_{2}$ \\
0 & 1 & 0 & 1 & 0 & $u \in C_{2}$ \\
\hline \hline
\end{tabular}

Покажем теперь, что $C_{2}$ порождает пустой граф. Допустим, что существуют несмежные вершины $u, v \in C_{2}$. Ясно, что $u, v \notin V F$. Обе вершины $u, v$ не смежны с вершинами $a, c, e$ (табл. 1). Каждая из вершин $u, v$ смежна по крайней мере с одной из вершин $b, d$. Пусть $u \sim d$. Так как $\{b, e, u, v\}$ не порождает $G_{1}$, то $b \nsim v$. Следовательно, $v \sim d$ и $u \nsim d$. Получаем, что $V F \cup\{u, v\}$ порождает $G_{6}$. Получили противоречие.

Итак, мы показали, что разбиение $C_{1} \cup C_{2} \cup C_{3}=V G$ является правильной 3раскраской графа $G$. Покажем теперь, что она является сильной.

Предположим теперь, что существуют несмежные вершины $u \in C_{1}$ и $v \in C_{3}$. Из табл. 1 ясно, что $u, v \notin V F, u \sim a, u \sim d, u \nsim b, v \sim b, v \sim e$ и $v \nsim d$. Допустим сначала, что и $c \nsim u$ и $c \nsim v$. Если $u \nsim е$ и $v \nsim a$, то $V F \cup\{u, v\}$ порождает $G_{7} . \mathrm{B}$ противном случае, в силу симметрии, можно считать, что $v \sim a$. Тогда $\{a, b, c, e, u, v\}$ порождает $G_{3}$ или $G_{4}$. Получаем противоречие. Поэтому можно считать, что $u \sim c$. Так как $\{c, d, u, v\}$ не порождает $G_{1}$, то $v \sim c$. Если $u \nsim e$ и $v \nsim a$, то $\{a, b, c, e, u, v\}$ порождает $G_{4}$. Поэтому можно считать, что $v \sim a$. Поскольку $\{a, d, e, u, v\}$ не порождает цикл $C_{5}$, то $u \sim e$. Итак, мы получаем порожденный подграф $F_{1}$, показанный на рис. 5 .

При наличии порожденного подграфа $F_{1}$ покажем, что $u \sim C_{2}$. Предположим, что существует вершина $w \in C_{2}$, не смежная с $u$. Ясно, что $w \in V G \backslash V F$. Так как $w$ не смежна с $a, c, e$ и $\{c, d, u, w\}$ не порождает $G_{1}$, то $w \sim d$.

Если $w \nsim v$, то $\{a, d, e, u, v, w\}$ порождает $G_{4}$; если $w \sim v$, то $\{a, d, u, v, w\}$ порождает $G_{5}$. Получаем противоречие.

Итак, доказано, что каждая вершина класса $C_{1}$ смежна со всеми вершинами класса $C_{3}$ или $C_{2}$. Аналогичное утверждение справедливо для любой вершины класca $C_{3}$.

Остается показать, что каждая вершина из $C_{2}$ смежна со всеми вершинами по 


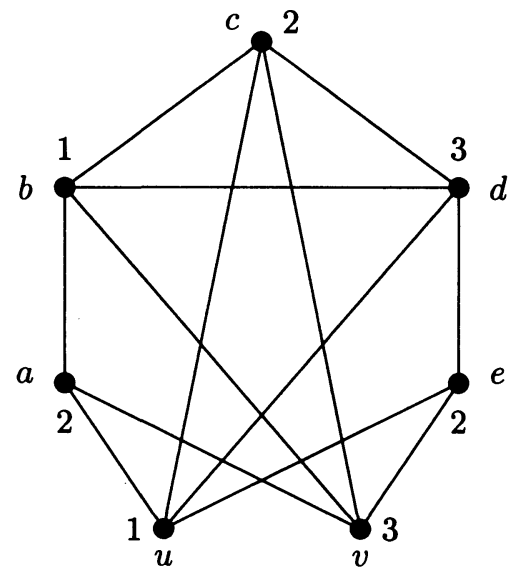

Рис. 5. Порожденный подграф $F_{1}$

крайней мере одного из классов $C_{1}$ или $C_{3}$. Предположим, что существуют вершины $u \in C_{2}, v \in C_{1}$ и $w \in C_{3}$ такие, что $u \nsim v$ и $v \nsim w$. Так как $v \in C_{1}$ и $v \nsim u \in C_{2}$, то, по доказанному выше, $v \sim C_{3}$. В частности, $v \sim w$. Пусть сначала $u=c$. Тогда $v, w \in V G \backslash V F$. Если $a \nsim w$ и $e \nsim v$, то $V F \cup\{v, w\}$ порождает $G_{7}$. В противном случае можно считать, что $a \nsim w$. Тогда $\{a, b, c, e, v, w\}$ порождает $G_{3}$ или $G_{4}$. Получаем противоречие.

Если $u=a$ (соответственно, $u=e$ ), то, согласно табл. $1, u$ смежна с каждой вершиной класса $C_{1}$ (соответственно, класса $C_{3}$ ). Поэтому остается рассмотреть случай, когда $u \in V G \backslash V F$. В соответствии с табл. 1 и в силу симметрии можно считать, что $u \sim d$. Тогда $w \in V G \backslash V F$. Пусть $v=b$, то есть $u \nsim b$. Так как $\{b, c, u, w\}$ не порождает $G_{1}$, то $w \nsim c$. Так как $\{a, b, u, w\}$ не порождает $G_{1}$, то $w \nsim a$. Тогда $V F \cup\{u, w\}$ порождает $G_{6}$. Получаем противоречие. Остается рассмотреть последний вариант $u \sim b$ и $v \in V G \backslash V F$. Так как $\{a, u, v, w\}$ и $\{e, u, v, w\}$ не порождают $G_{1}$, а $v \sim w$, то $a \nsim w$ и $e \nsim u$. Тогда $\{a, b, d, e, u, v, w\}$ порождает $G_{8}$. Опять получаем противоречие.

Таким образом, доказано, что $G$ имеет сильную 3 -раскраску, то есть $G \in S(3)$.

Лемма 6. Если граф $G$ удовлетворяет условиям леммы 4 и не содержст порожденного подграфа $F$ (рuс. 3), то $G \in S(3)$.

Доказательство. В силу леммы 4, мы можем считать, что $G$ содержит порожденный подграф $H$ (рис. 3). Зафиксируем в $G$ порожденный подграф $H$ вместе с обозначениями вершин, указанными на рис. 3 . Если $G=H$, то $G \in S(3)$. В противном случае пусть $u \in V G \backslash V H$. Как и в доказательстве леммы 5 , составим таблицу вариантов смежности вершины $u$ с вершинами множества $V H$ (табл. 2). Из таблицы 2 ясно, что возможны восемь вариантов $H_{1}, \ldots, H_{8}$ (рис.6) для подграфа, порожденного множеством $V H \cup\{u\}$.

Покажем, что вариант $H_{1}$ невозможен. Вершины $u$ и $c$ не являются раздвоенными. Поэтому существует вершина $v$ такая, что $v \sim u$ и $v \nsim c$. Так как $v \nsim c$, в соответствии с таблицей 2 , возможны три случая:

(1) $v \sim b$ и $v \sim d$ (тогда $\{b, d, u, v\}$ порождает $G_{2}$ ); 

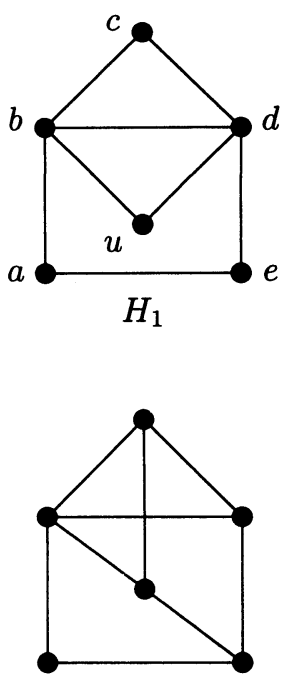

$\mathrm{H}_{5}$

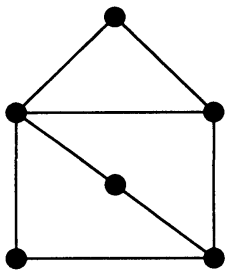

$\mathrm{H}_{2}$

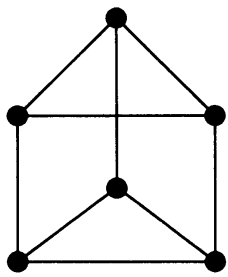

$H_{6}$

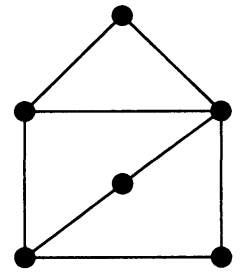

$\mathrm{H}_{3}$

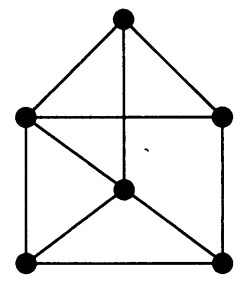

$\mathrm{H}_{7}$

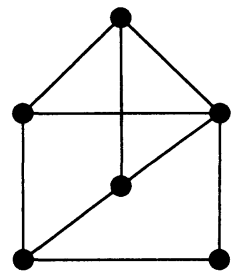

$\mathrm{H}_{4}$

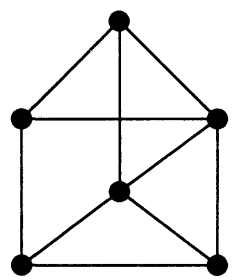

$\mathrm{H}_{8}$

Рис. 6.

Таблица 2.

\begin{tabular}{|c|c|}
\hline$a b c d e$ & Запрещенный порожденный подграф, или подграф $G(V H \cup\{u\})$ \\
\hline$* 000 *$ & $G(b, c, d, u)=G_{1}$ \\
\hline 10011 & $G(a, c, e, u)=G_{1}$ \\
\hline $110 * 1$ & $G(a, c, e, u)=G_{1}$ \\
\hline * 1100 & $G(b, c, e, u)=G_{1}$ \\
\hline $0011 *$ & $G(a, c, d, u)=G_{1}$ \\
\hline * $111 *$ & $G(b, c, d, u)=G_{2}$ \\
\hline * 1000 & $G(b, c, d, e, u)=F$ \\
\hline $0001 *$ & $G(a, b, c, d, u)=F$ \\
\hline 00101 & $G(a, b, c, d, u)=F$ \\
\hline * 0100 & $G(b, c, d, e, u)=F$ \\
\hline 01011 & $G(a, c, d, e, u)=F$ \\
\hline 11010 & $G(a, b, c, e, u)=F$ \\
\hline 01010 & $H_{1}$ \\
\hline 01001 & $H_{2}$ \\
\hline 10010 & $H_{3}$ \\
\hline 10101 & $H_{6}$ \\
\hline * 1101 & $H_{5}, H_{7}$ \\
\hline $1011 *$ & $H_{4}, H_{8}$ \\
\hline
\end{tabular}




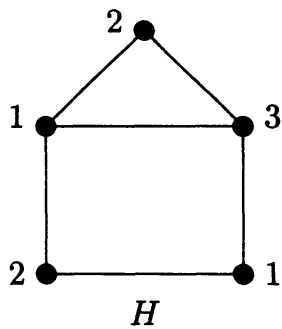

$H$

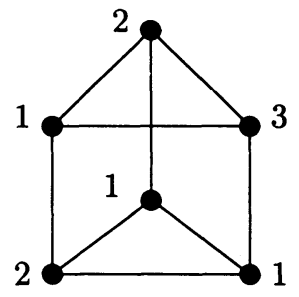

$H_{6}$

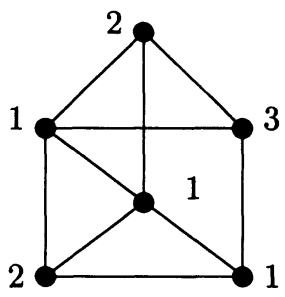

$\mathrm{H}_{7}$

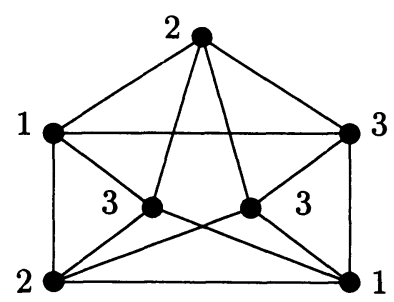

Рис. 7.

(2) $v \sim b$ и $v \sim e($ тогда $\{b, c, e, u, v\}$ порождает $F)$;

(3) $v \sim a$ и $v \sim d$ (тогда $\{a, c, d, u, v\}$ порождает $F$ ).

Покажем, что вариант $H_{2}$ (аналогично $H_{3}$ ) невозможен. В графе $H_{2}$ вершины $u$ и $a$ раздвоены. Поэтому существует вершина $v$, смежная с $u$ и не смежная с a. В соответствии с таблицей 2, возможны два варианта (случай, когда $V F \cup\{u\}$ порождает $H_{1}$ исключен):

(1) $v \sim e$ и $v \nsim c$ (тогда $\{c, e, u, v\}$ порождает $\left.G_{1}\right)$;

(2) $v \sim e$ и $v \sim c$ (тогда $\{a, c, e, u, v\}$ порождает $F$ ).

Покажем, что $H_{4}$ и $H_{5}$ невозможны. Так как в $H_{4}$ вершины $u$ и $b$ раздвоены, существует вершина $v$ такая, что $v \sim b$ и $v \nsim u$. Согласно таблице 2 и с учетом рассмотренных случаев, получаем, что $v \sim e$ и $v \sim c$. Тогда $\{c, b, e, u, v\}$ порождает $F$. Получаем противоречие.

Итак, мы показали, что для каждой вершины $u \notin V H$ множество $V H \cup\{u\}$ порождает один из графов $H_{6}, H_{7}$ или $H_{8}$. Если существуют две смежные вершины $u, v \notin V H$, то множество $\{a, e, u, v\}$ порождает $G_{2}$, что невозможно. Поэтому $V G \backslash V H$ является независимым множеством. Так как в $G$ нет раздвоенных вершин, для любых $u, v \in V G \backslash V H$ выполняется соотношение $N(u) \cap V H \neq N(u) \cap V H$.

Если $V H \cup\{u\}$ порождает $H_{7}$, а $V H \cup\{v\}$ порождает $H_{8}$, то $V H \cup\{u, v\}$ порождает $G_{10}$, что невозможно. Поэтому остается четыре варианта для графа $G$, показанные на рис. 7. Все они являются сильно 3-раскрашиваемыми графами, поэтому $G \in S(3)$. Лемма и теорема доказаны.

Следующий алгоритм вытекает из доказательства теоремы 1 и имеет сложность $O(m n)$, где $m$ и $n-$ число ребер и вершин графа соответственно.

\section{Алгоритм распознавания сильно 3-раскрашиваемых графов}

Вход: граф $G$.

Выход: $G \notin S(3)$ или $G \in S(3)$ и сильная 3 -раскраска $C_{1}, C_{2}, C_{3}$.

Шаг 1. Если граф $G$ двудольный и $C_{1}, C_{2}$ - разбиение его на доли, то выход $G \in$ $S(3)$ и $C_{1}, C_{2}, C_{3}=\varnothing$. Иначе перейти к шагу 2. 
Шаг 2. Пусть $R_{1}, \ldots, R_{k}$ - классы эквивалентности раздвоенных вершин графа $G$. Построить граф $G^{\prime}$, удаляя из $R_{i}$ все вершины, кроме одной, для любого $i=1, \ldots, k$. Для $X \subseteq V G^{\prime}$ положим

$$
R(X)=\cup\left\{R_{i}: X \cap R_{i} \neq \varnothing\right\} \subseteq V G .
$$

Шаг 3. Если граф $G^{\prime}$ содержит доминирующую вершину $d$ и граф $G^{\prime}-d$ двудольный с долями $G_{1}^{\prime}$ и $G_{2}^{\prime}$, то выход $G \in S(3)$ и $C_{1}=R\left(C_{1}^{\prime}\right), C_{2}=R\left(C_{2}^{\prime}\right)$, $C_{3}=R(\{d\})$. Если граф $G^{\prime}-d$ не двудольный, то выход $G \notin S(3)$. Если граф $G^{\prime}$ не содержит доминирующей вершины, то перейти к шагу 4.

Шаг 4. Если в $G$ нет треугольника, то выход $G \notin S(3)$. Иначе найти любой треугольник $C$ (пусть $V C=\{u, v, w\})$ и перейти к шагу 5 .

Шаг 5. Найти вершину $x \notin V G$, смежную ровно с одной вершиной из $C$ (пусть $x \sim w)$ и перейти к шагу 6. Если такой вершины нет, то выход $G \notin S(3)$.

Шаг 6. Найти вершину $y$, смежную ровно с одной из вершин $u, v$ и не смежную с $w$ и перейти к шагу 7. Если такой вершины нет, то выход $G \notin S(3)$.

Шаг 7. Если множество $U=\{u, v, w, x, y\}$ порождает граф $F$ (рис. 3), то по доказательству леммы 6 либо можно найти в $G$ запрещенный порожденный подграф (в этом случае выход $G \notin S(3)$ ), либо однозначно строится сильная 3-раскраска $C_{1}, C_{2}, C_{3}$ (в этом случае выход $G \in S(3)$ и $C_{1}, C_{2}, C_{3}$ ).

Шаг 8. Если $U$ порождает граф $H$ (рис. 3), то по доказательству леммы 6 либо находится запрещенный порожденный подграф (в этом случае выход $G \notin S(3)$ ), либо порожденный подграф $F$ (тогда переход к шагу 8 ), либо граф $G$ совпадает с одним из графов на рис. 7 (выход $G \in S(3)$ и $C_{1}, C_{2}, C_{3}$ - раскраска; указанная на рис. 7 ).

\section{Задача "Сильная $k$-раскрашиваемость"}

Вход: граф $G$ и целое $k \geqslant 1$.

Boпрос: справедливо ли включение $G \in S(k)$ ?

Предложение 1. Задача “Силъная $k$-раскрашиваемость" является NP-полной при любом $k \geqslant 4$.

Доказательство. Известно [1], что задача "Раскрашиваемость графа в $k$ цветов" $\mathrm{NP}$-полна при любом $k \geqslant 3$. Сведем ее к рассматриваемой задаче. Пусть задан граф $G$ и число $k \geqslant 3$. Построим граф $H$ путем добавления к $G$ изолированной вершины $u \in V G$. Покажем, что $G$ имеет правильную $k$-раскраску тогда и только тогда, когда $H \in S(k+1)$.

Если граф $G$ имеет правильную $k$-раскраску $C_{1}, \ldots, C_{k}$, то положим

$$
D_{1}=C_{1} \cup\{u\}, \quad D_{i}=C_{i}, \quad 2 \leqslant i \leqslant k, \quad D_{k+1}=\varnothing
$$

и получим сильную $(k+1)$-раскраску графа $H$, то есть $H \in S(k+1)$.

Если теперь граф $H$ имеет сильную $(k+1)$-раскраску $C_{1}, \ldots, C_{k+1}$, причем вершина $u \in C_{1}$, то для $u$ существует цветной класс $C_{i}, 2 \leqslant i \leqslant k+1$, такой, что $u \sim C_{i}$. Так как $u$ - изолированная вершина, то $C_{i}=\varnothing$ и граф $G$ является раскрашиваемым. 
Две $k$-раскраски графа считаются одинаковыми, если они различаются только обозначениями цветных классов. Соединение $G+H$ графов $G$ и $H$ с $V G \cap V H=\varnothing$ определяется как граф с множеством вершин $V G \cup V H$ и множеством ребер

$$
E G \cup E H \cup\{x y: x \in V G, y \in V H\} .
$$

Теорема 2. Граф $G \in S(3)$ имеет более одной силъной 3-раскраски тогда и толъко тогда, когда он является несвязным двудольным графом, либо может быть получен раздвоением вериин следующих графов: $K_{1}+B$, где $B-$ несвязный двудолъный граф; $\mathrm{P}_{4}, \mathrm{H}$ и $\mathrm{H}_{6}$ (puc. 7).

Доказательство. Докажем достаточность. Пусть $B$ - несвязный двудольный граф. Тогда $B$ имеет по крайней мере два различных разбиения на доли: $C_{1}, C_{2}$ и $D_{1}, D_{2}$. Если $G=B$ (соответственно, $G=K_{1}+B$ ), то $G$ имеет по крайней мере две различные сильные 3-раскраски: $C_{1}, C_{2}, C_{3}=\varnothing$ и $D_{1}, D_{2}, D_{3}=\varnothing$ (соответственно, $C_{1}, C_{2}, C_{3}=\{u\}$ и $D_{1}, D_{2}, D_{3}=\{u\}$, где $\left.\{u\}=V K_{1}\right)$. Легко проверить, что каждый из графов $P_{4}, H$ и $H_{6}$ имеет две различные сильные 3-раскраски. Наконец, очевидно, что если граф имеет более одной сильной 3-раскраски, то любой граф, полученный из него раздвоением вершин, также обладает таким свойством.

Докажем необходимость. Без ограничения общности будем считать, что в графе $G$ нет раздвоенных вершин. Пусть граф $G$ имеет две различные сильные 3раскраски: $C_{1}, C_{2}, C_{3}$ и $D_{1}, D_{2}, D_{3}$.

Свойство А. Любой цветной класс одной из указанных раскрасок имеет непустое пересечение не более, чем с двумя цветными классами другой раскраски.

Действительно, иначе вторая раскраска не будет сильной.

Из того, что раскраски различны, следует, что существуют вершины $u$ и $v$, имеющие одинаковый цвет в одной раскраске и различные цвета в другой раскраске. Пусть $u, v \in C_{1}, u \in D_{1}$ и $v \in D_{2}$.

Положим $A=C_{1} \cap D_{1} \neq \varnothing$ и $B=C_{1} \cap D_{2} \neq \varnothing$. Ясно, что $A \nsim B$ и, по свойству $\mathrm{A}$, $C_{1}=A \cup B$. Так как вершины $u$ и $v$ не являются раздвоенными, существует вершина $w$ такая, что $w \sim u$ и $w \nsim v$. В силу симметрии можно считать, что $w \in C_{2}$. Так как $v \in C_{1}, v \nsim w$ и $w \in C_{2}$, то $v \sim C_{3}$ (иначе раскраска $C_{1}, C_{2}, C_{3}$ не является сильной). Аналогично, $B \sim D_{3}$.

Свойство В. Справедливы соотношения $A \sim D_{3}$ и $B \sim D_{3}$.

Действительно, $A \subseteq D_{1}, A \nsim B, B \subseteq D_{2}$ и $A, B \neq \varnothing$, поэтому $A \sim D_{3}$ (иначе раскраска $D_{1}, D_{2}, D_{3}$ не является сильной). Аналогично, $B \sim D_{3}$.

Из $v \in B, v \nsim w$ и $B \sim D_{3}$ следует, что $w \notin D_{3}$. Так как $w \sim u$ и $u \in D_{1}$, то $w \notin D_{1}$. Поэтому $w \in D_{2}$ и множество $D=C_{1} \cap D_{2}$ не пусто.

Пусть $C=C_{2} \backslash D$. Ясно, что $C \cap D_{2}=\varnothing$ и $C \nsim D$. Из свойства А следует, что либо $C \subseteq D_{1}$, либо $C \subseteq D_{3}$.

Из $v \in D_{2}$ и $v \sim C_{3}$ следует, что $C_{3} \cap D_{2}=\varnothing$. Пусть $E=C_{3} \cap D_{3}$ и $F=C_{3} \cap D_{1}$. Ясно, что $E \nsim F$ и, по свойству $\mathrm{A}, C_{3}=E \cup F$. Так как $A, F \subseteq D_{1}$, то $A \nsim F$. Так как $B, D \subseteq D_{2}$, то $B \nsim D$. Из свойства В следует, что $A \sim E$. Из $u \nsim F$ и $u \in C_{1}$ следует, что $F \sim C_{2}$, то есть $F \sim C$ и $F \sim D$. Аналогично, $v \nsim D$ и $v \in C_{1}$, поэтому $D \sim C_{3}$. Далее, $w \nsim B$ и $w \in C_{2}$, откуда $B \sim C_{3}$.

Возможны два случая. Рассмотрим случай $F \neq \varnothing$. Так как $F \subseteq D_{1}$ и $F \sim C$, то $C \subseteq D_{3}$. Из свойства В следует, что $A \sim C$ и $B \sim C$. Так как $A \nsim F, A \subseteq C_{1}$ и 


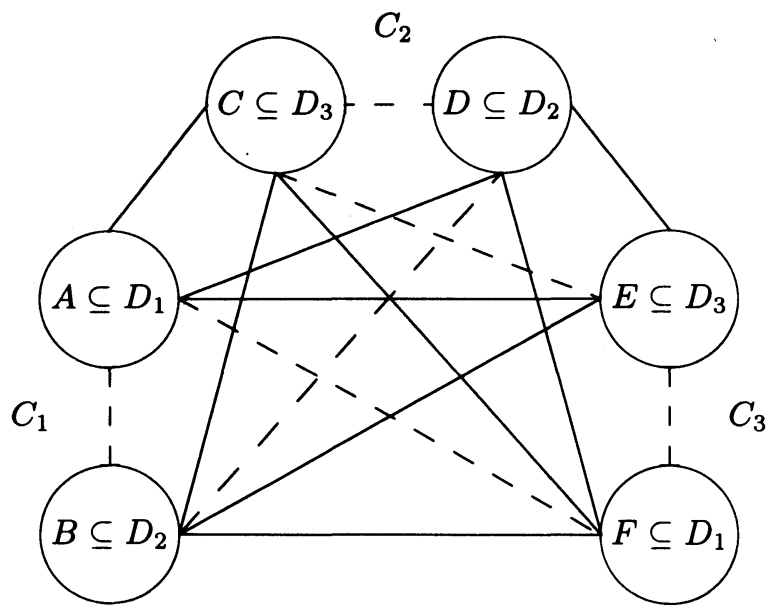

Рис. 8.

$F \subseteq C_{3}$, то $A \sim C_{2}$. Наконец, из $C, E \subseteq D_{3}$ следует, что $C \nsim E$. Мы получаем граф, показанный на рис. 8. Так как в $G$ нет раздвоенных вершин, то $G \in\left\{P_{4}, H, H_{6}\right\}$ и в этом случае теорема верна.

В случае $F=\varnothing$ рассмотрим сначала подслучай $C \subseteq D_{1}$ (возможно, $C=\varnothing$ ). Так как $A, C \subseteq D_{1}$, то $A \nsim C$. Из соотношений

$$
C \subseteq D_{1}, \quad D \subseteq C_{2}, \quad C \nsim D, \quad D \neq \varnothing
$$

следует, что $C \sim E \subseteq D_{3}$. Так как в $G$ нет раздвоенных вершин, то $|E| \leqslant 1$ и либо $G=B$, либо $G=K_{1}+B$, где $B=G(A \cup B \cup C \cup D)-$ несвязный двудольный граф.

Теперь рассмотрим подслучай $\varnothing \neq C \subseteq D_{3}$. По свойству В $A \sim C$ и $B \sim C$. Так как $C, E \subseteq D_{3}$, то $C \nsim E$. Так как $D \subseteq D_{2}, C \subseteq D_{3}, D \nsim C$ и $C \neq \varnothing$, то $D \sim D_{1}$. В частности, $D \sim A$. Итак, $G$ совпадает с графом, показанным на рис. 8, при $F=\varnothing$. Он получается из $P_{4}$ или $H$ раздвоением вершин (так как множество $E$ может быть пустым). Теорема доказана.

\section{Список литературы}

1. Гэри М., Джонсон Д., Вычислителъные машины и труднорешаемые задачи. Мир, Москва, 1982.

2. Brandstadt A., Partitions of graphs into one or two independent sets and cliques. Discrete Math. (1998) 152, 47-54.

3. Jensen T. R., Toft B., Graph coloring problems. Wiley, New York, 1995.

Статья поступила 13.04.1998. 